\title{
Invertebrados aquáticos associados a serapilheira de Salix humboldtiana em um riacho subtropical
}

\author{
Franko TelöKen ${ }^{1,3}$; Edélti F. Albertoni ${ }^{1, \varpi} ;$ Luiz U. Hepp $^{1,2} \&$ Cleber Palma-Silva $^{1}$ \\ 1. Programa de Pós-Graduação em Biologia de Ambientes Aquáticos Continentais, Instituto de Ciências Biológicas, Laboratório \\ de Limnologia, Universidade Federal do Rio Grande, Rio Grande, Brasil. 2. Programa de Pós-Graduação em Ecologia. \\ Universidade Regional Integrada do Alto Uruguai e das Missões, Laboratório de Biomonitoramento, Erechim, Brasil. 3. \\ Endereço atual: COMUSA - Serviços de Água e Esgoto de Novo Hamburgo, Novo Hamburgo, Brasil.
}

\begin{abstract}
Resumo. O material alóctone da mata ripária é fonte importante para a cadeia alimentar detritívora em riachos florestados. Este estudo teve como objetivos i) estimar a perda de massa foliar de Salix humboldtiana Willd., ii) analisar as alterações na composição química dos detritos durante o período de perda de massa, e iii) avaliar a assembleia de invertebrados colonizadoresem um córrego arenoso na planície costeira sul do Rio Grande do Sul (Brasil), com a hipótese de que os detritos de $S$. humboldtiana constituem habitat favorável a colonização por invertebrados, e que estes contribuem para sua degradação. Foram incubadas 28 bolsas de folhiço na superfície do sedimento do córrego, com retiradas em sete datas amostrais. Mudanças na composição química e a abundância, riqueza e grupos tróficos funcionais de invertebrados durante a perda de peso foram analisadas. Aos 184 dias foi registrada a perda de $70 \%$ da biomassa dos detritos $\left(\mathrm{k}=0.0066 \mathrm{dia}^{-1}\right)$. Foram registrados 14197 organismos, distribuídos em 36 táxons. Oligochaeta (43.6\%), Hydrobiidae (16.6\%), Chironominae (11.3\%) e Hydracarina (6.6\%) foram os mais representativos. O desenvolvimento do biofilme, observado por meio do incremento das concentrações de nitrogênio e fósforo totais nos detritos, pode ter favorecido a colonização por raspadores na fase final do experimento. A hipótese inicial foi corroborada em parte, pois considerando a baixa abundância de potenciais fragmentadores, a contribuição da comunidade de invertebrados para a degradação dos detritos é reduzida, sendo os coletores - catadores o grupo trófico funcional mais abundante da assembleia.
\end{abstract}

[Palavras-chave: decomposição, riacho arenoso, detritívoros, grupos tróficos funcionais]

Aвstract. Aquatic invertebrates associated with Salix humboldtiana litter in a subtropical stream: Allochthonous material from riparian forest is an important source for detritivore food chain in streams. This study aimed to: i) estimate the leaf mass loss of Salix humboldtiana Willd., ii) analyze the changes in the detritus chemical composition during mass loss, and iii) analyze the invertebrate colonizers assemblage of a sandy stream in south coastal plain of the Rio Grande do Sul state (Brazil). We hypothesized that S. humboldtiana debris constitute favorable habitat for colonization by invertebrates, which in turn contribute to debris degradation. Twenty eight bags were incubated on the sediment surface of the stream in seven sampling dates. During the decomposition process we analyzed changes of detritus chemical composition and the abundance, richness and functional trophic groups of invertebrates. After 184 days we recorded an average loss of $70 \%$ of debris $\left(\mathrm{k}=0.0066\right.$ day $\left.^{-1}\right)$ and counted a total of 14197 invertebrate individuals, distributed in 36 taxa. Oligochaeta (43.6\%), Hydrobiidae (16.6\%), Chironominae $(11.3 \%)$ and Hydracarina $(6.6 \%)$ were the most representative. The biofilm development, observed through increased concentrations of total phosphorus and nitrogen in the debris, may have favored colonization by scrapers at the end of the decomposition period. The initial hypothesis was partially corroborated as the abundance of potential shredders was low, the contribution of invertebrates assemblage to litter decomposition was low, and the gathering-collectors were the most abundant group.

[Keywords: decomposition, sandy stream, detritivores, functional trophic groups]

\section{INTRODUÇÃO}

O aporte de matéria orgânica alóctone constitui fontede energia e nutrientes para a biota em ecossistemas lóticos sombreados (Vanotte et al. 1980; Webster \& Benfield 1986). Nestes ambientes, a cadeia de detritos representa a principal via de circulação de matéria e energia (Begon et al. 2007). A entrada de matéria orgânica alóctone, a retenção e a decomposição dos detritos constituem processos fundamentais para o metabolismo de riachos de pequeno porte (Abelho 2001;

\section{Editor asociado: Fernando Unrein}

$\triangle$ dmbefa@furg.br
Minshall \& Rugenski 2006). Em linhas gerais, o processamento do detrito orgânico passa por uma fase de lixiviação dos compostos solúveis, condicionamento microbiano e fragmentação por invertebrados aquáticos (Webster \& Benfield 1986).

A comunidade de invertebrados aquáticos desempenha importante papel nos ecossistemas de água doce, participando das teias alimentares como presas para peixes, anfíbios e aves (Albertoni \& Palma-Silva 2010). 
Em adição, a associação dos invertebrados aquáticos aos detritos orgânicos e o seu processamento tem sido reportados por estudos em diversos ecossistemas (ver revisão de Graça 2001). Embora esta associação ainda não seja completamente clara, sabe-se que os detritos podem prover alimento, abrigo ou ambos para estes organismos (Dudgeon \& Wu 1999; Graça 2001; Janke \& Trivinho-Strixino 2007). Assim, os invertebrados aquáticos atuam sobre o detrito orgânico auxiliando na transformação da matéria orgânica particulada grossa (MOPG) em matéria orgânica particulada fina (MOPF) (Webster \& Benfield 1986).

Os estudos sobre o papel dos invertebrados no processamento dos detritos em ambientes subtropicais ainda são insipientes. Ainda assim, alguns estudos reportam que uma abordagem interessante no estudo da associação de invertebrados em detritos vegetais é a análise funcional de sua alimentação baseada nos mecanismos morfocomportamentais de aquisição e consumo de alimentos (Cummins et al. 2005; Silva et al. 2010; Telöken et al. 2011, entre outros). Entre os recursos utilizados, a MOPG e MOPF constituem fontes alimentares para os fragmentadores e coletores que colonizam os detritos vegetais em decomposição (Capello et al. 2004; Graça \& Cressa 2010; Biasi et al. 2013). Ainda podem se associar aos detritos, organismos raspadores, utilizando-se do perifíton aderido, e os predadores que predam sobre os outros grupos (Cummins et al. 2005; Merritt \& Cummins 2006).

Embora a planície costeira sul do Brasil apresente cerca de $40 \%$ de sua superfície com ambientes aquáticos, estudos sobre os processos ecossistêmicos nestes ainda são escassos. A região costeira do extremo sul do Brasil apresenta uma ampla área de terras baixas, sendo os ambientes lagunar-lacustre os mais predominantes (Vieira \& Rangel 1988), em sua maior parte ocupada pelo sistema lagunar Patos-Mirim (Villwock \& Tomazelli 1995). A unidade mórfica básica é a restinga, originada a partir de acumulações sedimentares continentais e marinhas tendo como constituição básica as areias (Vieira \& Rangel 1988). Como consequência das baixas declividades, os riachos apresentam reduzida correnteza. Em períodos com baixa precipitação pluviométrica, ocorre perda significativa da força hidráulica acarretando em um intenso processo de acumulação de sedimentos no leito do canal, originando bancos arenosos (Vieira \& Rangel 1988).
A espécie arbórea Salix humboldtiana Willd. (Salicaceae) desenvolve-se nas margens de cursos d'água e em ambientes com água subterrânea em abundância, nas Américas do Sul e Central (Backes \& Irgang 2002; Carvalho 2003). Esta espécie é constituinte comum da vegetação ripária de lagos e riachos arenosos típicos da planície costeira do Rio Grande do Sul (Batista et al. 2007; Trindade et al. 2010). Salix humboldtiana apresenta elevada produção de serapilheira foliar, sendo responsável por importante aporte de matéria orgânica e nutrientes aos ambientes adjacentes onde se desenvolve (Aceñolaza et al. 2010). Estudos com decomposição de $S$. humboldtiana são escassos destacando-se Leguizamon et al. (1992), Capello et al. (2004) e Poi de Neiff et al. (2006), desenvolvidos no sistema hidrológico do rio Paraná, Argentina, e Telöken et al. (2011) em um lago raso no sul do Brasil. Para a planície costeira sul do Brasil, a literatura sobre o processo de decomposição desta espécie em riachos é inexistente, apesar de sua abundância e ampla distribuição como constituinte da vegetação ripária nos ambientes aquáticos. Considerando a abundância de S. humboldtiana junto aos riachos arenosos da planície costeira do extremo Sul do Brasil, este estudo tem como objetivos i) estimar a perda de massa foliar de $S$. humboldtiana em um riacho arenoso, ii) analisar as alterações na composição química durante o período de perda de massa e iii) estimar a densidade, riqueza e composição funcional da assembleia de invertebrados colonizadores ao longo do tempo. A hipótese central do estudo é de que os detritos de $S$. humboldtiana constituem habitat favorável a colonização por invertebrados aquáticos, e que estes contribuem através dos fragmentadores para a degradação foliar desta espécie.

\section{Material e Métodos}

\section{Área de estudo}

O estudo foi desenvolvido em um riacho localizado na região costeira do Rio Grande do Sul (3209'27" Se $52^{\circ} 11^{\prime} 18^{\prime \prime} \mathrm{O}$ ). O clima é descrito como Cfa (subtropical úmido) de acordo com a classificação de Köppen. A região encontra-se em uma faixa de transição entre condições tropicais e temperadas com temperaturas médias anuais entre 15 e $18{ }^{\circ} \mathrm{C}$ e precipitação pluviométrica anual variando de 1200 a $1500 \mathrm{~mm}$ (SEPLAG 2012). A área de drenagem do riacho é composta por campos, banhados e mata ripária composta basicamente pelas espécies arbóreas Salix humboldtiana Willd., Erythrina crista-galli L., Ficus organensis Miq., Sebastiania commersoniana (Baillon) L. B. Smith \& R. J. Downs, Erytroxylum argentinum O. E. Schulz, entre outras (Batista et al. 2007). 
O arroio Bolaxa é semelhante a maioria dos arroios da planície costeira sul do Brasil, com baixa declividade e movimento lento da água (Vieira \& Rangel 1988). O substrato do riacho estudado é basicamente composto por areia e eventualmente ocorre o desenvolvimento de estandes de macrófitas aquáticas, entre elas Potamogeton pectinatus L., Salvinia auriculata Aubl., Myriophyllum brasiliensis Camb. (Batista et al. 2007). Durante o período de realização do experimento o $\mathrm{pH}$ da água permaneceu próximo à neutralidade (6.70 \pm 0.38$)$, enquanto que a condutividade elétrica e alcalinidade total foram elevadas $(245.5 \pm 22.7 \mu \mathrm{S} /$ $\mathrm{cm}$ e $1373.1 \pm 135.1 \mathrm{meq} / \mathrm{L} \mathrm{CO}_{2}$, respectivamente). Os teores de oxigênio dissolvido variaram de 1.48 a $7.38 \mathrm{mg} / \mathrm{L}$, porém, esta oscilação é normal para os riachos da região em épocas de estiagem (Tagliani et al. 1992). As concentrações de nutrientes e clorofilaa mantiveram-se baixas durante todo o período (Nitrogênio total: $0.85 \pm 0.41 \mathrm{mg} / \mathrm{L}$; Fósforo total: $0.04 \pm 0.03 \mathrm{mg} / \mathrm{L}$; Clorofila-a: $1.74 \pm 1.90 \mu \mathrm{g} / \mathrm{L})$.

\section{Experimento de campo e procedimentos em laboratório}

O experimento foi realizado entre agosto/2009 e fevereiro/2010. Foram utilizadas folhas senescentes de $S$. humboldtiana coletadas às margens do riacho estudado. No laboratório, as folhas foram secas a temperatura ambiente $\left(20 \pm 2{ }^{\circ} \mathrm{C}\right)$ durante duas semanas. Foram confeccionadas 28 bolsas de folhiço com telas de nylon e polietileno, de dimensões $20 \times 30 \mathrm{~cm}$ e aberturas de malha de $0.1 \mathrm{~cm}^{2}$ na face inferior (em contato com o sedimento) e $1.0 \mathrm{~cm}^{2}$ na face oposta (adaptado de Bedford 2004). Em cada bolsa foi adicionado $6.0 \pm 0.1 \mathrm{~g}$ de folhas. As bolsas foram dispostas no fundo do riacho presas com fios de nylon entre si e a tijolos para que ficassem submersas. Após 1, 7, 20, 50, 90, 126 e 184 dias de incubação, quatro bolsas de detritos foram retiradas aleatoriamente do riacho. Os detritos remanescentes foram lavados em água corrente, sobre peneira de $250 \mu \mathrm{m}$, sendo posteriormente secos em estufa $\left(60^{\circ} \mathrm{C} / 48 \mathrm{~h}\right)$ para determinação do peso seco remanescente.

Após lavagem e remoção dos organismos, os detritos secos foram triturados e submetidos a análises químicas quanto às concentrações de nitrogênio total (NT; Kjeldahl; Allen et al. 1974), fósforo total (PT) (Fassbender 1973; Baumgarten \& Rocha 1996), matéria orgânica (incineração $550^{\circ} \mathrm{C} /$ 4 h) (Wetzel \& Likens 1991) e polifenóis (adaptado de Bärlocher \& Graça 2005). As concentrações de carbono foram estimadas multiplicando os valores de matéria orgânica por 0.465 (Westlake 1965). As concentrações químicas foram expressas em porcentagem por peso seco de detrito (\%PS), com exceção de polifenóis (U.D.O./g PS).

Os invertebrados retidos na peneira (malha de $250 \mu \mathrm{m}$ ) foram fixados em etanol $80 \%$ e identificados em um estereomicroscópio até o menor nível taxonômico possível utilizando chaves taxonômicas específicas (Thorp \& Covich 1991; Elmoor-Loureiro 1997; Buckup \& Bond-
Buckup 1999; Merritt et al. 2008; Mugnai et al. 2010). Foi determinada a riqueza e abundância de táxons, considerando-se como táxon o grupo identificado, conforme corrente uso na literatura com abordagem de avaliação da comunidade de invertebrados associada a detritos (Yamamuro \& Lamberti 2007; Resende et al. 2010; Moulton et al. 2010, entre outros). A densidade de organismos foi expressa como número de indivíduos por grama de peso seco de detrito (indivíduos/g PS). Para determinar os padrões de sucessão de organismos em relação ao uso dos recursos, os invertebrados foram classificados em grupos tróficos funcionais (GTF) desde que bem estabelecido em literatura para o nível taxonômico utilizado no presente estudo, de acordo com classificação proposta por Callisto et al. (2001), Cummins et al. (2005) e Merritt et al. (2008). Os invertebrados foram depositados na Coleção de Invertebrados Límnicos Subtropicais do Laboratório de Limnologia da Universidade Federal do Rio Grande.

\section{Análises de dados}

Ao final do período estudado foi determinada a taxa de perda foliar (k), utilizando o coeficiente exponencial negativo proposto por Webster \& Benfield (1986) respectivamente com as equações $\% \mathrm{R}=\left(\mathrm{W}_{\mathrm{t}} / \mathrm{W}_{0}\right) * 100$ (para porcentagem de peso remanescente) e $k=-\ln (\% R / 100) / t$, onde $W_{t}$ é o peso seco no tempo $t$ (em dias) e $W_{0}$ é o peso seco inicial. Foram avaliadas as diferenças entre as concentrações dos compostos químicos do detrito ao longo do tempo por meio de análise de variância (ANOVA-one way) seguida por pós-teste de Tukey (HSD), considerando significativo $P<0.05$. Os valores de riqueza e densidade dos grupos taxonômicos ao longo do tempo foram analisados por meio de análise de variância (ANOVA-one way) seguida por pós-teste de Tukey (HSD), considerando significativo $P<0.05$.

\section{Resultados}

\section{Perda de massa foliar}

Ao final do período estudado foi determinado o peso remanescente de $69.48 \pm 3.81 \%$ representando um coeficiente de perda de massa de $\mathrm{k}=0.0066 \pm 0.0007 \mathrm{dia}^{-1}$ (Figura 1). Em $24 \mathrm{~h}$ foram lixiviados $32.16 \pm 2.86 \%$ do peso inicial dos detritos, representando $\mathrm{k}=0.3908 \pm 0.0437 \mathrm{dia}^{-1}$ (Figura 1).

\section{Análises químicas dos detritos}

As concentrações de matéria orgânica dos detritos mantiveram-se sempre superiores a $90 \%$ durante todo o período de estudo, porém com variações significativas ao longo do tempo (Tabela 1). As concentrações de polifenóis apresentaram rápida redução até o $20^{\circ}$ dia. As concentrações de nitrogênio total 
Tabela 1. Variação na composição química da serapilheira de Salix humboldtiana Willd., entre agosto/2009 e fevereiro/ 2010, em um córrego arenoso da planície costeira do Rio Grande do Sul (Brasil). Tempo zero=folhas senescentes nãoincubadas. Médias \pm desvios-padrões de nitrogênio total (\%NT), fósforo total (\%PT), matéria orgânica (\%MO), carbono $(\% \mathrm{C})$ e concentrações de polifenóis (U.D.O./g PS). N:P, relação nitrogênio:fósforo, C:N, relação carbono:nitrogênio, C: $\mathrm{P}$, relação carbono fósforo. Letra sdiferentes $(\mathrm{a}-\mathrm{f})$ representam diferenças significativas $(P<0.05)$.

Table 1. Changes in the chemical composition of Salix humboldtiana Willd. leaf litter, between august 2009 and February 2010 , in a sandy stream of coastal plain of Rio Grande do Sul (Brazil). Time- $0=$ senescent leaves not incubated. Mean \pm SD of total nitrogen $(\% \mathrm{NT})$, total phosphorus $(\% \mathrm{PT})$, organic matter $(\% \mathrm{MO})$, carbon $(\% \mathrm{C})$ and polyphenol concentration (U.D.O./g PS). N:P, nitrogen:phosphorus relationship; C:N, carbon:nitrogen relationship; C:P, carbon:phosphorus relationship. Different letters (a-f) represent significant difference $(P<0.05)$.

\begin{tabular}{|c|c|c|c|c|c|c|c|c|}
\hline $\begin{array}{l}\text { Tempo } \\
\text { (dias) }\end{array}$ & $\%$ NT & $\%$ PT & $\% \mathrm{MO}$ & $\% \mathrm{C}$ & Polifenóis (U.D.O./g PS) & $\mathrm{N}: \mathrm{P}$ & $C: N$ & $\mathrm{C}: \mathrm{P}$ \\
\hline 0 & $0.98 \pm 0.04^{a}$ & $0.08 \pm 0.00$ & $94.96 \pm 0.22^{a c}$ & $44.63 \pm 0.10^{a c}$ & $13.53 \pm 0.41^{a}$ & $12: 1$ & $46: 1$ & $558: 1$ \\
\hline 1 & $1.65 \pm 0.38^{b c}$ & $0.09 \pm 0.05$ & $96.29 \pm 0.14^{b}$ & $45.26 \pm 0.06^{b}$ & $10.72 \pm 0.45^{a}$ & $18: 1$ & $27: 1$ & $503: 1$ \\
\hline 7 & $1.40 \pm 0.06^{a b}$ & $0.11 \pm 0.00$ & $95.29 \pm 0.11^{a}$ & $44.79 \pm 0.05^{a}$ & $4.27 \pm 0.24^{b}$ & $13: 1$ & $32: 1$ & $407: 1$ \\
\hline 20 & $1.69 \pm 0.07^{b c}$ & $0.17 \pm 0.01$ & $94.18 \pm 0.11^{c d}$ & $44.27 \pm 0.05^{c d}$ & $1.76 \pm 0.03^{c}$ & $10: 1$ & $26: 1$ & $260: 1$ \\
\hline 50 & $1.93 \pm 0.09^{b c}$ & $0.11 \pm 0.00$ & $94.21 \pm 0.20^{c d}$ & $44.28 \pm 0.09^{c d}$ & $1.53 \pm 0.07 d f$ & $18: 1$ & $23: 1$ & $403: 1$ \\
\hline $\begin{array}{c}90 \\
126\end{array}$ & $\begin{array}{l}2.37 \pm 0.08^{c} \\
2.19 \pm 0.05^{c}\end{array}$ & $\begin{array}{l}0.11 \pm 0.01 \\
0.10 \pm 0.01\end{array}$ & $\begin{array}{l}94.72 \pm 0.27^{a c} \\
93.85 \pm 0.08^{d}\end{array}$ & $\begin{array}{l}44.52 \pm 0.13^{a c} \\
44.11 \pm 0.04^{d}\end{array}$ & $\begin{array}{l}2.83 \pm 0.07^{d} \\
1.86 \pm 0.06^{e}\end{array}$ & $\begin{array}{l}22: 1 \\
22: 1\end{array}$ & $\begin{array}{l}19: 1 \\
20: 1\end{array}$ & $\begin{array}{l}405: 1 \\
441: 1\end{array}$ \\
\hline 184 & $2.19 \pm 0.04^{c}$ & $0.13 \pm 0.02$ & $90.92 \pm 0.03^{e}$ & $42.73 \pm 0.02^{e}$ & $1.97 \pm 0.07^{f}$ & $17: 1$ & $20: 1$ & $329: 1$ \\
\hline
\end{tabular}

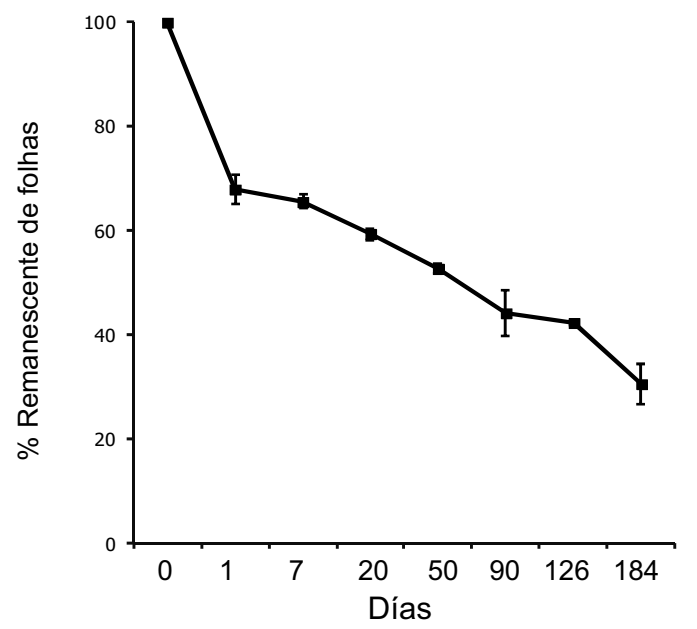

Figura 1. Perda de massa da serapilheira de Salix humboldtiana Willd. (em porcentagem de peso remanescente) no período entre agosto/2009 e fevereiro/ 2010 em um córrego arenoso, planície costeira do Rio Grande do Sul (Brasil).

Figure 1. Mass loss of Salix humboldtiana Willd. leaf litter (percentage of remaining weight) in the period between August/2009 and February/2010 in a sandy stream, coastal plain of Rio Grande do Sul (Brazil).

apresentaram incremento significativo ao longo do tempo, enquanto as concentrações de fósforo total não apresentaram variações ao longo do experimento.

\section{Invertebrados associados}

Foram contabilizados 14197 organismos distribuídos em 36 grupos taxonômicos (Tabela 2). Os mais representativos foram Oligochaeta (43.60\%), Hydrobiidae (16.63\%), Chironominae (11.26\%), Hydracarina $(6.61 \%)$, Ostracoda $(4.80 \%)$ e Orthocladiinae $(4.69 \%)$, totalizando $87.60 \%$ da assembleia. Em 24 horas, a densidade média de invertebrados foi $11.41 \pm 1.58$ individuos/g PS (Tabela 2). Foi observado aumento da densidade até o $20^{\circ}$ dia (524.16 \pm 83.61 indivíduos/g PS), com posterior diminuição destes valores, chegando a $40.99 \pm 17.13$ indivíduos/g PS no $126^{\circ}$ dia.

No primeiro dia de colonização foram registrados 16 táxons colonizando os detritos (Tabela 2). No $7^{\circ}$ dia foi registrada a maior riqueza (29 táxons) e posterior redução até o final do experimento ( $184^{\circ}$ dia com 15 táxons).

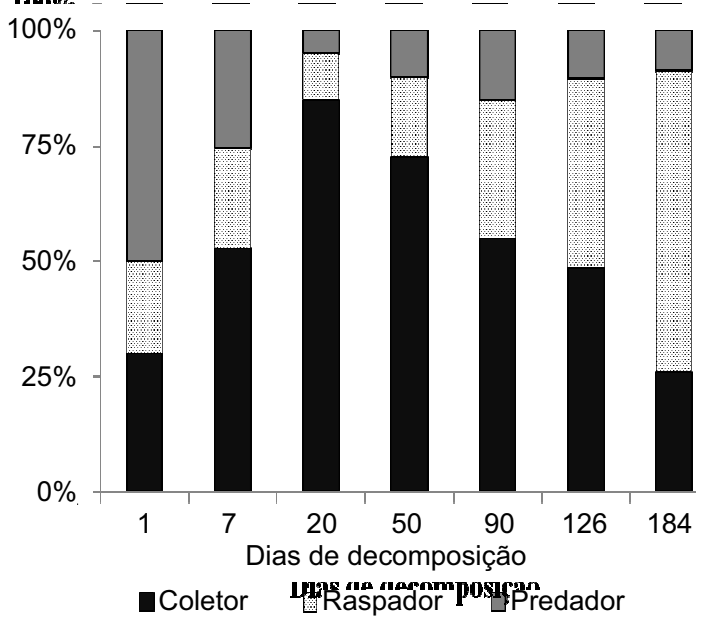

Figura 2. Distribuição em porcentagem dos grupos tróficos funcionais dos invertebrados associados a serapilheira de Salix humboldtiana Willd., entre agosto/2009 e fevereiro/ 2010, em um córrego arenoso da planície costeira do Rio Grande do Sul, excluindo Chironomidae, e considerado Hyallelidae como coletor.

Figure 2. Percentage distribution of functional feeding groups associated with Salix humboldtiana Willd. leaf litter, between august 2009 and February 2010, in a sandy stream, coastal plain of Rio Grande do Sul (Brazil), excluding Chironomidae, Hyallelidae as colector. 
Tabela 2. Invertebrados associados a serapilheira de Salix humboldtiana Willd., entre agosto/2009 e fevereiro/2010, em um córrego arenoso da planície costeira do Rio Grande do Sul (Brasil). Densidades médias (indivíduos/g PS), abundâncias (\%), riqueza (estimada para os táxons identificados) e grupos tróficos funcionais (GTF) dos táxons em cada período de amostragem. Pr: predador, Co-Fi: coletor - filtrador, Co-Ca: coletor-catador, Ra: raspador, Fr: fragmentador. Letras diferentes representam diferenças significativas $(a-c)(P<0.05)$.

Table 2. Invertebrates associated with Salix humboldtiana Willd. leaf litter, between August/2009 and February /2010 in a sandy stream of coastal plain of Rio Grande do Sul (Brazil). Mean densities (individuos/g DW) abundance $(\%)$, richness (calculated for all taxa) and functional trophic groups (FTG) of taxain each sampling period. Pr: predators, Co-Fi: filtering-collectors, Co-Ca: gathering-collectors, Ra: scrapers, Fr: shredders. Different letters represent significant differences $(\mathrm{a}-\mathrm{c})(P<0.05)$.

\begin{tabular}{|c|c|c|c|c|c|c|c|c|c|}
\hline \multirow[t]{2}{*}{ Taxa } & \multirow{2}{*}{ GTF } & \multicolumn{7}{|c|}{ Densidades Médias (ind/g PS) } & \multirow[t]{2}{*}{$\%$} \\
\hline & & 1 & 7 & 20 & 50 & 90 & 126 & 184 & \\
\hline Rhizopoda & $\operatorname{Pr}$ & 0 & 7.49 & 154.52 & 0 & 0 & 0 & 1799.27 & 0.76 \\
\hline Porifera & $\mathrm{Co}-\mathrm{Fi}$ & 0 & 0 & 0 & 0 & 0 & 15.02 & 0 & 0.01 \\
\hline Tricladida & $\mathrm{Co}-\mathrm{Ca} / \mathrm{Pr}$ & 0 & 14.32 & 0 & 0 & 8.33 & 0 & 0 & 0.02 \\
\hline Nemertea & $\operatorname{Pr}$ & 0 & 0 & 0 & 8.80 & 0 & 0 & 0 & 0.01 \\
\hline Nematoda & $\mathrm{Co}-\mathrm{Ca}$ & 0 & 157.51 & 149.73 & 530.86 & 293.87 & 290.51 & 743.83 & 1.42 \\
\hline Hirudinea & $\operatorname{Pr}$ & 0 & 7.27 & 53.96 & 297.84 & 66.67 & 60.33 & & 0.38 \\
\hline Oligochaeta & $\mathrm{Co}-\mathrm{Ca}$ & 33.69 & 4224.37 & 34182.02 & 8797.37 & 1294.42 & 808.84 & 1205.19 & 43.60 \\
\hline Hydracarina & $\operatorname{Pr}$ & 345.06 & 3063.49 & 1604.85 & 1117.92 & 427.50 & 150.97 & 1353.37 & 6.61 \\
\hline \multicolumn{10}{|l|}{ Cladocera } \\
\hline Chydoridae & $\mathrm{Co}-\mathrm{Ca}$ & 106.68 & 990.85 & 581.73 & 17.24 & 0 & 0 & 28.88 & 1.66 \\
\hline Daphniidae & $\mathrm{Co}-\mathrm{Fi}$ & 0 & 40.98 & 0 & 8.62 & 0 & 0 & 0 & 0.05 \\
\hline Macrothricidae & $\mathrm{Co}-\mathrm{Ca}$ & 0 & 6.83 & 0 & 0 & 0 & 0 & 0 & 0.01 \\
\hline \multicolumn{10}{|l|}{ Amphipoda } \\
\hline \multicolumn{10}{|l|}{ Isopoda } \\
\hline Sphaeromatidae & Co-Ca & 0 & 7.00 & 0 & 0 & 0 & 0 & 0 & 0.01 \\
\hline Cyclopoida & $\mathrm{Co}-\mathrm{Ca} / \mathrm{Pr}$ & 0 & 779.69 & 306.32 & 17.61 & 0 & 0 & 0 & 1.07 \\
\hline Harpacticoida & $\mathrm{Co}-\mathrm{Ca} / \mathrm{Pr}$ & 0 & 14.53 & 31.15 & 8.62 & 0 & 0 & 0 & 0.05 \\
\hline Ostracoda & $\mathrm{Co}-\mathrm{Ca}$ & 6.81 & 905.57 & 1268.08 & 163.67 & 58.58 & 15.02 & 7288.32 & 4.80 \\
\hline \multicolumn{10}{|l|}{ Collembola } \\
\hline Entomobryidae & $\mathrm{Co}-\mathrm{Ca}$ & 6.74 & 36.51 & 32.53 & 18.87 & 82.64 & 0 & 57.76 & 0.17 \\
\hline \multicolumn{10}{|l|}{ Ephemeroptera } \\
\hline Baetidae & $\mathrm{Co}-\mathrm{Ca} / \mathrm{Ra}$ & 6.43 & 0 & 30.44 & 132.04 & 8.33 & 45.05 & 0 & 0.17 \\
\hline Caenidae & $\mathrm{Co}-\mathrm{Ca} / \mathrm{Ra}$ & 34.19 & 815.38 & 479.61 & 155.90 & 125.13 & 75.21 & 34.72 & 1.55 \\
\hline \multicolumn{10}{|l|}{ Odonata } \\
\hline Coenagrionidae & $\operatorname{Pr}$ & 6.81 & 34.33 & 8.31 & 0 & 0 & 0 & 0 & 0.05 \\
\hline \multicolumn{10}{|l|}{ Coleoptera } \\
\hline Elmidae & $\mathrm{Co}-\mathrm{Ca} / \mathrm{Ra}$ & 0 & 0 & 7.62 & 0 & 33.46 & 136.36 & 0 & 0.09 \\
\hline Staphyli & $\operatorname{Pr}$ & 13.55 & 21.01 & 0 & 0 & 0 & 75.76 & 34.72 & 0.08 \\
\hline Hydrophilidae & $\mathrm{Co}-\mathrm{Ca} / \mathrm{Pr}$ & 0 & 51.96 & 156.34 & 35.21 & 0 & 0 & 0 & 0.22 \\
\hline \multicolumn{10}{|l|}{ Chironomidae } \\
\hline Tanypodinae & $\mathrm{Co}-\mathrm{Ca} / \mathrm{Pr}$ & 6.81 & 35.46 & 53.55 & 8.47 & 0 & 45.05 & 0 & 0.12 \\
\hline Orthocladiinae & $\begin{array}{c}\mathrm{Co}-\mathrm{Ca} / \mathrm{Fr} \\
/ \mathrm{Pr} / \mathrm{Ra} \\
\mathrm{Co}-\mathrm{Ca} /\end{array}$ & 143.55 & 779.45 & 3201.26 & 1042.18 & 12.56 & 45.05 & 0 & 4.69 \\
\hline Chironominae & $\begin{array}{c}\mathrm{Co}-\mathrm{Fi} / \mathrm{Fr} / \\
\mathrm{Pr} / \mathrm{Ra}\end{array}$ & 231.70 & 803.52 & 5064.96 & 5628.82 & 864.98 & 1273.82 & 185.13 & 11.26 \\
\hline Pupas & & 29.79 & 78.32 & 425.50 & 466.19 & 45.90 & 15.02 & 0 & 0.90 \\
\hline Ceratopogonidae & $\begin{array}{c}\mathrm{Co}-\mathrm{Ca} / \mathrm{Pr} \\
\text { / } \mathrm{Ra}\end{array}$ & 0 & 7.27 & 22.82 & 17.42 & 0 & 0 & 0 & 0.04 \\
\hline Simuliidae & Co-Fi & 0 & 0 & 32.17 & 431.20 & 0 & 45.05 & 0 & 0.39 \\
\hline Sciomyzidae & $\operatorname{Pr}$ & 0 & 7.27 & 0 & 0 & 0 & 0 & 0 & 0.01 \\
\hline Muscidae & $\operatorname{Pr}$ & 0 & 0 & 0 & 0 & 8.33 & 0 & 0 & 0.01 \\
\hline Trichoptera & & & & & & & & & \\
\hline $\begin{array}{l}\text { Hidroptilidae } \\
\text { Gastropoda }\end{array}$ & $\mathrm{Co}-\mathrm{Ca} / \mathrm{Ra}$ & 0 & 21.01 & 0 & 34.55 & 31.23 & 0 & 28.57 & 0.08 \\
\hline Ampullaridae & $\mathrm{Ra}$ & 0 & 0 & 16.27 & 27.67 & 0 & 0 & 0 & 0.04 \\
\hline Ancylidae & $\mathrm{Ra}$ & 44.78 & 646.66 & 559.93 & 650.58 & 16.67 & 120.26 & 666.57 & 2.01 \\
\hline Hydrobiidae & $\mathrm{Ra}$ & 80.54 & 1930.27 & 3501.72 & 1526.06 & 850.70 & 851.74 & 23385.46 & 16.63 \\
\hline Planorbidae & $\mathrm{Ra}$ & 0 & 63.91 & 148.05 & 51.58 & 33.46 & 15.02 & 84.03 & 0.30 \\
\hline Bivalvia & $\mathrm{Co}-\mathrm{Fi}$ & 0 & 0 & 0 & 0 & 0 & 0 & 180.91 & 0.07 \\
\hline Densidade total (ind/g PS) & & $11.41^{\mathrm{a}}$ & $157.42^{b c}$ & $524.16^{c}$ & $213.54^{a b c}$ & $42.71^{\mathrm{ab}}$ & $40.99 \mathrm{ab}$ & $370.77^{b c}$ & \\
\hline Riqueza & & $16^{\mathrm{ab}}$ & $29^{\mathrm{ab}}$ & $26^{\mathrm{a}}$ & $27^{\mathrm{ab}}$ & $19^{\mathrm{b}}$ & $19^{\mathrm{ab}}$ & $15^{\mathrm{ab}}$ & \\
\hline
\end{tabular}


Os coletores-catadores representaram a maior parcela da assembleia (52.8\%), sendo $70 \%$ destes Oligochaeta. Os raspadores representaram $30 \%$, sendo $80 \%$ destes Hydrobiidae. Os predadores representaram $11.6 \%$, potenciais fragmentadores 3\% e coletores-filtradores $2.6 \%$. Excluindo os potenciais fragmentadores (Chironomidae), considerando Hyallelidae como coletor, e agrupando coletores catadores e filtradores como um grupo, a representação dos GTF ao longo do processo de degradação é mostrada na Figura 2.

\section{Discussão}

O coeficiente de perda de massa estimado no presente trabalho foi menor do que os valores obtidos em estudos com Salix humboldtiana em diferentes ambientes no sistema hidrológico do Rio Paraná (Argentina). Os coeficientes de decomposição de $S$. humboldtiana foram maiores em um pântano ( $\mathrm{k}=0.019 /$ dia) (Poi de Neiff et al. 2006), e em um canal da planície aluvial ( $\mathrm{k}=0.0101 / \mathrm{dia})$ (Capello et al. 2004) e em um rio ( $\mathrm{k}=0.0119 / \mathrm{dia})$ (Leguizamon et al. 1992). As taxas de degradação variam de acordo com a composição química da água, qualidade do detrito, ação do fluxo da correnteza e da ação de invertebrados, em especial fragmentadores. No entanto, acredita-se que a perda de massa mais lenta em relação aos estudos citados tenha ocorrido por efeitos da dinâmica do sedimento do riacho. O fundo arenoso é instável a mudanças no fluxo, o que acarreta em suspensão do mesmo e posterior deposição sobre o detrito, retardando o processo de perda de massa e dificultando a colonização por invertebrados. Detritos enterrados apresentam menores taxas de degradação do que os dispostos na superfície do sedimento, sendo isto causado principalmente pela diminuição da abrasão física com a água em movimento, baixas concentrações de oxigênio dissolvido e limitação na ação de invertebrados (Herbst 1980; Smock et al. 1989; Metzler \& Smock 1990).

A perda de massa neste estudo foi rápida durante as primeiras horas devido à lixiviação de compostos solúveis. O material remanescente, composto por polissacarídeos estruturais é mais resistente à degradação, sendo sua perda de peso mais lenta (Bianchini Jr. 1999). Polifenóis também são compostos facilmente lixiviados do detrito (Hepp et al. 2009). Provavelmente a rápida lixiviação dos polifenóis nos primeiros 20 dias de decomposição tenha se tornado um fator facilitador para a colonização microbiana e por invertebrados.

O condicionamento microbiano é importante para o processo de aumento da palatabilidade do detrito, o que facilita a colonização do mesmo por parte dos invertebrados detritívoros (Graça 2001; Graça et al. 2001; Wantzen et al. 2008). A variação na concentração de NT e PT dos detritos ao longo do tempo reflete o condicionamento microbiano do detrito, o qual diminui as relações C:N e C:P refletindo o aumento do valor nutricional dos detritos (Graça \& Cressa 2010). Neste estudo, o incremento de nutrientes, em especial nitrogênio, é um forte indicador do condicionamento microbiano do detrito (Lester et al. 1994) pois é reconhecido que o aumento de nitrogênio com o tempo de degradação vegetal favorece a atuação dos decompositores (fungos e bactérias) (Brum \& Esteves 2001).

Aliado ao aumento na concentração de nitrogênio, a diminuição nas concentrações de polifenóis favorece também a colonização por invertebrados, pois proporcionam um incremento na qualidade do detrito. Recentes estudos sugerem que altas concentrações de compostos secundários nos detritos (polifenóis) inibem a colonização de invertebrados retardando o processo de fragmentação (Wantzen et al. 2008; Trevisan \& Hepp 2007; Hepp et al. 2009; Biasi et al. 2013). No presente estudo, foi observada a redução da relação C:N e C:P em cerca de $50 \%$ e do conteúdo de polifenóis em cerca de $70 \%$ durante o período de degradação, o que refletiu na melhoria da qualidade do detrito e, consequentemente, na densidade de invertebrados colonizadores.

Os coletores catadores representaramos organismos mais abundantes na assembleia de invertebrados, e aos 20 dias foram dominados por Oligochaeta, que chegou a $65 \%$ de abundância na assembleia. A dominância de coletores-catadores em detritos é associada à acumulação de MOPF originado do próprio detrito, ou de partículas orgânicas em suspensão na coluna d'água que acumulam na superfície dos substratos (Richardson 1992; Grubbs et al. 1995; Serra et al. 2013). A abundância de raspadores no final do período do experimento, representados principalmente por Hidrobiidae, pode estar relacionada com o incremento nas concentrações de NT e PT no detrito com o decorrer da decomposição, o que 
provavelmente favoreceu o estabelecimento do perifíton associado à MOPF.

Embora o nível de identificação taxonômica não permita categorizar funcionalmente com precisão alguns grupos (e.g., Chironomidae), para a grande maioria a classificação trófica funcional é bem estabelecida em literatura. Os organismos de Amphipoda apresentaram abundância muito baixa durante o processo de degradação (menos de 1\%), e foram todos pertencentes a família Hyallelidae (Hyallela curvispina), reportada por Capello et al. (2004) e Wantzen \& Wagner (2006) como coletores, embora Cummins et al. (2005) e Zilli et al. (2008) categorizem esta família como fragmentadora. Neste trabalho adotamos a classificação dos dois primeiros autores. Outros potenciais fragmentadores seriam alguns gêneros de Chironomidae, não identificados no presente estudo. Cummins et al. (2005) categorizam a família Chironomidae como coletores-catadores em vários riachos de Mata Atlântica no Brasil. Conforme colocam Wantzen \& Wagner (2006), em geral as espécies descritas como fragmentadores para a região neotropical apresentam baixa biomassa, e provavelmente têm menor influência sobre a degradação dos detritos comparada a regiões temperadas. Biasi et al. (2013), em recente estudo sobre Chironomidae associados a decomposição foliar em riachos subtropicais reportam que apenas $2.2 \%$ dos gêneros foram classificados como fragmentadores, Zilli et al. (2008) reportam dois gêneros em diferentes mesohabitats aquáticos da planície de inundação do rio Paraná (Argentina) pertencentes a este grupo trófico e Silva et al. (2010) reportam apenas um gênero de Chironomidae fragmentador em um lago próximo ao riacho estudado.

Assim, com base na baixa abundância de potenciais fragmentadores registrada durante a degradação de $S$. humboldtiana, pode ser considerado que este grupo funcional não contribuiu de forma significativa para a velocidade de degradação dos detritos. Os fragmentadores consistem no principal GTF que contribui para a degradação da matéria orgânica particulada grossa em regiões temperadas (Wallace \& Webster 1996; Graça et al. 2001; Serra et al. 2013). No entanto, estudos em regiões tropicais e subtropicais têm registrado baixas abundâncias destes organismos colonizando os detritos (Dobson et al. 2002; Stripari \& Henry 2002; Gonçalves et al. 2006, 2007; Carvalho \&Uieda 2009; Poi de Neiff et al. 2009; Telöken et al. 2011; Biasi et al. 2013). Irons et al. (1994) propõem que a importância defragmentadores paraoprocesso de decomposição é maior em ambientes temperados, enquanto que nos ambientes tropicais e subtropicais os microrganismos possuem maior importância. Os resultados referentes a assembléia de invertebrados e sua categorização funcional no riacho estudado ainda são insipientes, e ressaltam a importância de estudos mais detalhados sobre a categorização funcional dos organismos encontrados. Futuros experimentos devem ser conduzidos para definir principalmente a real participação de fragmentadores no processo de degradação foliar, de forma a aplicar ou modificar as classificações propostas em outros trabalhos em diferentes ecossistemas nas várias regiões geográficas.

Considerando a composição química dos detritos de $S$. humboldtiana, a redução da concentração de polifenóis e das proporções $\mathrm{C}: \mathrm{N}$ e $\mathrm{C}: \mathrm{P}$ ao longo do tempo, sugere-se que estes detritos melhoraram a qualidade ao longo do processo de degradação, favorecendo a colonização por invertebrados e corroborando parte da hipótese inicial de nosso estudo. Por outro lado, mesmo com a categorização taxonômica utilizada, constatouse que o número de potenciais fragmentares foi reduzido ao longo do processo de perda de massa foliar, refutando parte de nossa hipótese de contribuição efetiva destes para a velocidade de degradação de $S$. humboldtiana. Embora a identificação taxonômica não tenha possibilitado identificar gêneros de Chironomidae potenciais fragmentadores, a participação em termos de abundância destes organismos ao longo do período experimental sempre foi inferior aos principais grupos tróficos (predomínio de coletores nas fases iniciais e raspadores na fase final), o que ressalta a importância da MOPF como recurso alimentar para estes organismos. O papel da MOPF no estabelecimento de comunidades de invertebrados em riachos foi recentemente demonstrado por Callisto \& Graça (2013), onde concluem que Chironomidae foi fortemente relacionado com as zonas deposicionais onde é maior a quantidade de MOPF. Por outro lado, a dinâmica do sedimento dos riachos arenosos subtropicais, condicionado às variações climáticas sazonais, pode atuar como fator regulador do processamento da matéria orgânica alóctone.

Agradecimentos: os autores agradecem aos técnicos do Laboratório de Limnologia/FURG, a CAPES, pela bolsa de estudos do primeiro autor, e aos dois revisores anônimos pela sugestões para melhoria do manuscrito. Luiz U. Hepp recebeapoio financeiro da FAPERGS (Proc. 12/1354-0) e 
CNPq (Proc. 471572/2012-8). Cleber Palma-Silva recebe apoio financeiro da FAPERGS (Proc. 10/1013455).

\section{REFERÊNCIAS}

AвELHO, M. 2001. From litterfall to breakdown in streams: a review. The Scientific World, 1:656-680.

AceÑolaZa, PG; LP ZAMBOnI; EE RodRIGUEZ \& JF Gallardo. 2010. Litterfall production in forests located at the predelta area of the Paraná River (Argentina). AFS, 67:1-10.

Albertoni, EF \&C Palma-Silva. 2010. Caracterização e importância dos invertebrados de águas continentais com ênfase nos ambientes de Rio Grande. Cadernos de Ecologia Aquática, 5:9-27.

Allen, SE; HM Grimshaw; JA Parkinson \& C Quarmby. 1974. Chemical analysis of ecological materials. Oxford, Blackwell Scientific Publications. Pp. 565.

Backes, P \& B Irgang. 2002. Árvores do sul: guia de identificação e interesse ecológico. $1^{\circ}$ ed. Porto Alegre, Clube da Árvore, Instituto Souza Cruz. Pp. 326.

BÄrLOCHER, F \& MAS GRAÇA. 2005. Total phenolics. In: GraÇA, MAS; F BÄrLocher \& MO GESSNER (eds.). Methods to study litter decomposition. Pp. 97-100. Springer, Berlin/ New York.

Batista, TL; RCA CANTEIRo; LPP Dorneles \& IG Colares. 2007. Levantamento florístico das comunidades vegetais na Área de Proteção Ambiental da Lagoa Verde, Rio Grande, RS. Rev. Brasil. Bioc., 5:225-227.

BAUMgARTEN, MGZ \& JMB Rocha. 1996. Manual de análises em oceanografia química. Rio Grande, Editora da FURG. Pp. 132.

Begon, M; CR TownSEnd \& JL HaRper. 2006. Ecologia: de indivíduos a ecossistemas. $4^{\mathrm{a}}$ ed. Porto Alegre, Artmed. Pp. 752.

BEDFORD, AP. 2004. A modified litter bag design for use in lentic habitats. Hydrobiologia, 529:187-193.

BIANCHIN JR,I.1999. Aspectos do processo de decomposição nos ecossistemas aquáticos continentais. In: Pompêo, MLM (ed.). Perspectivas da limnologia no Brasil. São Luís, Gráfica e Editora União. Pp. 21-43.

Biasi, C; AM Tonin; RM Restello \& LU Hepp. 2013. The colonisation of leaf litter by Chironomidae (Diptera): The influence of chemical quality and exposure duration in a subtropical stream. Limnologica, 43:427-433.

Brum, P \& FA Esteves. 2001. Dry weight loss and chemical change in the detritus of three tropical aquatic macrophytes species (Eleocharis interstincta, Nymphaea ampla and Potamogeton stenostachys) during decomposition. Acta Limnol. Brasil, 13:61-73.

BucKUP, L \& G Bond-BuckUp. 1999. Os crustáceos do Rio Grande do Sul. Porto Alegre. Editora Universidade/ UFRGS. Pp. 503.

Callisto, M; P Moreno \& FAR Barbosa. 2001. Habitat diversity and benthic functional trophic groups at Serra do Cipó, Southeast Brazil. Rev. Brasil. Biol., 61:259-266.

Callisto, M \& MAS Graça. 2013. The quality and availability of fine particulate organic matter for collector species in headwater streams. Internat. Rev. Hydrobiol., 98:132-140.

Capello, S; M Marchese \& I Ezcurra De Drago. 2004 Descomposición y colonización por invertebrados de hojas de Salix humboldtiana en la llanura aluvial del Río Paraná Medio. Amazoniana, 18:125-143.

Carvalho, EM \& VS Uieda. 2009. Diet of invertebrates sampled in leaf-bags incubated in a tropical headwater stream. Zoologia, 26:694-704.

CARVAlHo, PER. 2003. Espécies arbóreas brasileiras. Brasília, Embrapa Informação Tecnológica; Colombo, Paraná: Embrapa Florestas. Pp. 1039.

Cummins, KW; RW Merritt \& PCN Andrade. 2005. The use of invertebrates functional groups to characterize ecosystem attributes in selected streams and rivers in south Brazil. Stud. Neotrop. Fauna Environ., 40:69-89.

Dobson, M; A Magana; JM Mathooko \& FK NDEgwa. 2002. Detritivors in Kenyan highland streams: more evidence for the paucity of shredders in tropics? Freshw. Biol., 47:909-919.

DudGeON, D \& KKY Wu. 1999. Leaf litter in a tropical stream: food or substrate for macroinvertebrates? Archiv. Hydrobiol., 146:65-82.

ELMOOR-LoureIro, LMA. 1997.Manual de identificação de cladóceros límnicos do Brasil. Brasília, Editora Universa. Pp 155.

FAssbender, HW. 1973.Simultane P-Bestimmung in N-Kjeldahl-aufschlubb von Bodenproben. Die Phosphorsäure, 30:44-53.

Gonçalves Jr, JF; JS França; AO Medeiros; CA Rosa \& M CALLISTO. 2006. Leaf breakdown in a tropical stream. Internat. Rev. Hydrobiol., 91:164-177.

Gonçalves Jr, JF; MAS GRaÇA \& M Callisto. 2007. Litter decomposition in a cerrado savannah stream is retarded by leaf toughness, low dissolved nutrients and a low density of shredders. Freshw. Biol., 52:1440-1451

GraÇA, MAS. 2001. The role of invertebrates on leaf litter breakdown in a stream - a review. Intern. Rev. Hydrobiol., 86:383-393.

Graça, MAS; C Cressa; M Gessner; M Feio; K Callies; et AL. 2001. Food quality, feeding preferences, survival and growth of shredders from temperate and tropical streams. Freshw. Biol., 46:947-957.

Graça, MAS \& C Cressa. 2010. Leaf quality of some tropical and temperate tree species as food resource for stream shredders. Intern. Rev. Hydrobiol., 95:27-41.

GruBbS, SA; RE JACOBSEN \& KW CUMmINS. 1995. Colonization by Chironomidae (Insecta, Diptera) on three distinct leaf substrates in an Appalachian mountain stream. Ann. Limnol., 31:105-118.

Hepr, LU; R Delanora \& A Trevisan. 2009. Compostos secundários durante a decomposição foliar de espécies arbóreas em um riacho do sul do Brasil. Acta Bot. Brasil., 23:407-413.

HerbST, GN. 1980. Effects of burial on food value and consumption of leaf detritus by aquatic invertebrates in a lowland forest stream. Oikos, 35:411-424.

Irons, JG; MW Oswood; RJ Stout \& CM PRINGLe. 1994. Latitudinal patterns in leaf litter breakdown: is temperature really important? Freshw. Biol., 32:401411.

JANKe, H \& S TRIVINHO-StRIXINO. 2007. Colonization of leaf litter by aquatic macroinvertebrates: a study in a low order tropical stream. Acta Limnol. Brasil, 19:109-115.

Leguizamon, M; J Hammerly; MA Maine; N Suñe, \& MJ Pizzaro. 1992. Decomposition and nutrient liberation rates of plant material in the Parana medio River (Argentina). Hydrobiologia, 230:157-164.

Lester, PJ; SF Mitchell \& D Scott. 1994. Willow leaf and periphyton chemical composition, and the feeding preferences of Olinga feredayi (Trichoptera: Conoesucidae). New Zealand Journal of Marine and Freshwater Research, 28:13-18.

Magurran, AE. 2004. Measuring Biological Diversity. Oxford, Blackwell Publishing. Pp. 256. 
MerRITT, RW \& KW Cummins. 2006. Trophic relationships of macroinvertebrates. In: Lamberti, G \& R Hauer (eds.). Methods in Stream Ecology, $2^{\text {nd }}$ ed., Oxford, Elsevier. Pp. 585- 601.

Merritt, RW; KW Cummins \& MB Berg. 2008. An introduction to the aquatic insects of North America. Dubuque, Kendall/Hunt Publishing Co. Pp. 1214.

Metzler, GM \& LA SMOCK. 1990. Storage and dynamics of subsurface detritus in a sand-bottomed stream. Can. J. Fish. Aquat. Sci., 47:588-594.

MinSHALL, GW \& A RUGENSKI. 2006. Riparian processes and interactions. In: Lamberti, G \& R Hauer (eds.). Methods in Stream Ecology, $2^{\text {nd }}$ ed, Oxford, Elsevier. Pp. 721-742.

Moulton, TP; SAP Magalhães-Fraga; EF Brito \& FA BARBOSA. 2010. Macroconsumers are more important than specialist macroinvertebrate shredders in leaf processing in urban forest streams of Rio de Janeiro, Brazil. Hydrobiologia, 638:55-66.

Mugnai, R; JL Nessimian \& DF Baptista. 2010. Manual de Identificação de Macroinvertebrados Aquáticos do Estado do Rio de Janeiro. Rio de Janeiro: Technical Books. Pp. 176.

Poi de Neiff, A; JJ Neiff \& SL Casco. 2006. Leaf litter decomposition in three wetland types of the Paraná River flood plain. Wetlands, 26:558-566.

Poi de Neiff, A; ME Galassi \& MC Franceschini. 2009. Invertebrate assemblage associated with leaf litter in three floodplain wetlands on the Parana river. Wetlands, 29:896-906.

Rezende, RS; JF Gonçalves Jr \& MM Petrucio. 2010. Leaf breakdown and invertebrate colonization of Eucalyptus grandis (Myrtaceae) and Hirtella glandulosa Chrysobalanaceae) in two Neotropical lakes. Acta Limnol. Brasil, 22:23-34.

RichARDSON, JS. 1992. Food, microhabitat, or both? Macroinvertebrate use of leaf accumulations in a montane stream. Freshw. Biol., 27:169-176.

Seplag - Secretaria do Planejamento, Gestão e Participação Cidadã do estado do Rio Grande do Sul. 2012. Atlas Sócio-Econômico do Rio Grande do Sul. Disponível em: http://www.seplag.rs.gov.br/atlas. Acesso em: 23/12/2012.

SERRA, MN; R Albariño \& V D VillanUeVA. 2013. Invasive Salix fragilis alters benthic invertebrate communities and litter decomposition in northern Patagonian streams. Hydrobiologia, 701:173-188.

Silva, JS; WT Silveira; EF Albertoni \& C Palma-Silva. 2010. Diversity of Chironomidae (Diptera) during the decomposition of Nymphoides indica (L.) Kuntze in two subtropical lakes with different trophic levels (south Brazil). Panamjas, 5:557-571.

Smock, L; GM Metzler \& J GLAdDen. 1989. Role of debris dams in the structure and functioning of low-gradient head water streams. Ecology, 70:764-775.

Stripari, N de L \& R Henry. 2002. The invertebrate colonization during decomposition of Eichhornia azurea Kunth in a lateral lake in the mouth zone of
Paranapanema river into Jurumirim reservoir (São Paulo, Brazil). Braz. J. Biol., 62:293-310.

Tagliani, PRA; E Barbieri, \& AC Neto. 1992. About a sporadic phenomenon of fish mortality by environmental hypoxia in the Senandes streamlet, State of Rio Grande do Sul, Brazil. Ciênc. Cult., 44:404-406.

TelöKen, F; EF Albertoni \& C Palma-Silva. 2011. Leaf degradation of Salix humboldtiana Willd. (Salicaceae) and invertebrate colonization in a subtropical lake (Brazil). Acta Limnol. Brasil, 23(1):30-41.

THORP, JH \& AP Covich. 1991. Ecology and classification of North American freshwater invertebrates. New York, Academic Press Inc. Pp. 911.

Trevisan, A \& LU Hepp. 2007. Dinâmica de componentes químicos vegetais e fauna associada ao processo de decomposição de espécies arbóreas em um riacho do norte do Rio Grande do Sul. Neotrop. Biol. Conserv., 2: 54-60.

Trindade, CRT; SA Pereira; EF Albertoni \& C PalmaSILVA. 2010. Caracterização e importância das macrófitas aquáticas com ênfase nos ambientes límnicos do campus Carreiros - FURG, Rio Grande, RS. Cadernos de Ecologia Aquática, 5:1-22.

Vanotte, RL; GW Minshall; KW Cummins; JR Sedell \& CE Cushing. 1980. The River Continuum Concept. Can. J. Fish. Aquat. Sci., 37:817-822.

VIeIRA, EF \& SS Rangel. 1988. Planície costeira do Rio Grande do Sul: Geografia física, vegetação e dinâmica sóciodemográfica. Porto Alegre, Sagra. Pp. 256.

Villwock, JA \& LJ Tomazelli. 1995. Geologia Costeira do Rio Grande do Sul. Centro de Estudos de Geologia Costeira e Oceânica. Universidade Federal do Rio Grande do Sul. Porto Alegre. Notas técnicas, 8:1-45.

Wallace, JB \& JR Webster. 1996. The role of macroinvertebrates in stream ecosystem function. Ann. Rev. Entomol., 41:115-139.

Wantzen, KM; CM Yule; JM Mathooko \& C Pringle. 2008. Organic matter processing in tropical streams. In: Dudgeon, D (ed.). Tropical Stream Ecology. San Diego, Elsevier. Pp. 44-65.

WEBSTER, JR \& EF BENFIELD. 1986.Vascular plant breakdown in freshwater ecosystems. Ann. Rev. Ecol. Evol. Syst., 17: 567-94.

WeSTLAKE, DF. 1965. Some basic data for investigations of the productivity of aquatic macrophytes. J. Mem. Ist. Ital. Idrobiol., 18:229-248.

WetZeL, RG \& GE LiKENs. 1991. Limnological analyses. New York: Springer Publishers. Pp. 391.

YAMAMURO, AM \& GA LAMBERTI. 2007. Influence of organic matter on invertebrate colonization of sand substrata in a northern Michigan stream. J. N. Am. Benthol. Soc., 26:244-252.

Zilli, FL; L Montalto \& MR Marchese. 2008. Benthic invertebrate assemblages and functional feeding groups in the Paraná River floodplain (Argentina). Limnologica, 38:159-171. 